\title{
A!
}

This is an electronic reprint of the original article.

This reprint may differ from the original in pagination and typographic detail.

Rajamaki, Robin; Koivunen, Visa

\section{Near Field Active Imaging Using Sparse Arrays}

Published in:

Conference Record of the 52nd Asilomar Conference on Signals, Systems and Computers, ACSSC 2018

DOI:

10.1109/ACSSC.2018.8645096

Published: 01/01/2018

Document Version

Peer reviewed version

Please cite the original version:

Rajamaki, R., \& Koivunen, V. (2018). Near Field Active Imaging Using Sparse Arrays. In M. B. Matthews (Ed.), Conference Record of the 52nd Asilomar Conference on Signals, Systems and Computers, ACSSC 2018 (Vol. 2018-October, pp. 348-353). [8645096] IEEE. https://doi.org/10.1109/ACSSC.2018.8645096

This material is protected by copyright and other intellectual property rights, and duplication or sale of all or part of any of the repository collections is not permitted, except that material may be duplicated by you for your research use or educational purposes in electronic or print form. You must obtain permission for any other use. Electronic or print copies may not be offered, whether for sale or otherwise to anyone who is not an authorised user. 


\title{
Near Field Active Imaging Using Sparse Arrays
}

\author{
Robin Rajamäki and Visa Koivunen \\ Department of Signal Processing and Acoustics \\ School of Electrical Engineering, Aalto University, 02150 Espoo, Finland \\ Email: robin.rajamaki@aalto.fi, visa.koivunen@aalto.fi
}

\begin{abstract}
Sensor arrays designed for far field operation may experience performance degradation when imaging near field objects. Specifically, sparse active arrays utilizing the additional degrees of freedom provided by the sum co-array are susceptible to these effects, as the co-array depends on both the range and direction of scatterers close to the array. Consequently, a uniform far field sum co-array may become non-uniform in the near field. As a result, co-array processing algorithms, such as image addition, are subject to undesired grating lobes in the presence of near field scatterers. This paper proposes an extension to image addition for mitigating such undesired distortions. The method compensates for near field effects by computing spatially varying transmit and receive element weights. These weights minimize the discrepancy between the desired and achieved near field point spread function, while using as few image addition components as possible. Given a desired point spread function and a set of calibration measurements of the near field array steering vectors, a regularized convex optimization problem is then solved for each pixel of the image.
\end{abstract}

\section{INTRODUCTION}

Sparse sensor arrays can achieve comparable performance to conventional filled arrays, despite using only a fraction of the number of elements and RF front ends. For example, a sparse array of $N$ elements can match the far field point spread function (PSF) of a uniformly spaced array with $\mathcal{O}\left(N^{2}\right)$ elements [1], or resolve $\mathcal{O}\left(N^{2}\right)$ sources in direction finding applications [2], [3]. This is enabled by a virtual array called the co-array [4], which is constructed using vector sums or differences of element positions. For instance, the sum coarray arises in active sensing applications, including far field coherent imaging and radar [1], [5]. In the near field however, the underlying plane wave assumption in co-array processing is no longer valid. Hence, the co-array becomes spatially varying. This spatially varying co-array depends on both the focus range and look direction of the array [6]-[8]. Consequently, near field scatterers distort the PSF, when applying beamforming weights designed for far field operation. This distortion can be compensated for by adjusting the weights in the near field.

A straightforward approach to near field compensation is to correct for the wavefront curvature by multiplying the far field array weights by appropriate phase and amplitude factors [9], [10]. These factors are determined by the array geometry and assumed scatterer positions, which need to be either known or estimated a priori. This restriction may be overcome by decomposing the desired beampattern into spherical harmonics, and computing the near field element weights based on a finite harmonical series approximation [11]-[13]. Other works formulate (often convex) optimization problems, which minimize the discrepancy between the desired and realized beampatterns, while satisfying certain design constraints imposed on the pattern's mainlobes, sidelobes or nulls [14]-[17]. Most of the aforementioned works address the near field compensation problem from the perspective of passive (receive-only) arrays, whereas active (transceiving) arrays are of interest in this work. Furthermore, these works do not jointly consider near field compensation and co-array processing techniques, such as image addition [1]. Image addition synthesizes a desired PSF by adding together component images acquired using multiple sets of elements weights. These weights may be obtained from the SVD of the co-array weight matrix [18]. The rank of this matrix determines the number of component images, $Q$. In case of a phased array, $Q$ determines the number of transmissions, or time, required for image acquisition [19].

This paper develops a novel near field compensation method in the context of the sum co-array model and the image addition method. The main contributions of the paper are twofold. Firstly, we demonstrate that sparse arrays can be sensitive to near field effects, as their co-arrays contain fewer redundancies than uniform arrays. Secondly, we formulate a low-rank matrix retrieval problem for optimizing the near field element weights. This formulation utilizes calibration measurements of the near field array steering vectors to approximate a desired beampattern, while imposing a matrix rank constraint to keep the number of component images low. Since the optimization problem is non-convex and generally difficult to solve exactly, we propose two convex relaxations to it. We evaluate the performance of the proposed approaches in simulation, where we consider imaging a scene of coherent near field scatterers. The suggested near field compensation methods show improved image quality over the uncompensated case, even when only few noisy narrowband calibration measurements are available.

The paper is organized as follows. Section II reviews the signal model. Section III describes how near field scatterers distort an array's PSF, and motivates the need for mitigating such effects. Section IV formulates the near field compensation problem, and section $\mathrm{V}$ presents the proposed solutions. Section VI demonstrates the performance of these solutions in numerical simulations, and section VII concludes the paper.

\section{SIGNAL MODEL}

Let an active linear array with $N_{\mathrm{t}}$ transmitters and $N_{\mathrm{r}}$ receivers be located on the $x^{\prime}$-axis at $\mathcal{D}_{\mathrm{t}}=\left\{d_{\mathrm{t}, m} \in \mathbb{R}\right\}_{m=1}^{N_{\mathrm{t}}}$ and $\mathcal{D}_{\mathrm{r}}=\left\{d_{\mathrm{r}, n} \in \mathbb{R}\right\}_{n=1}^{N_{\mathrm{r}}}$. The array is used to image a collection of $K$ coherent point reflectors. Fig. 1 illustrates the setup, showing a single transmitter (Tx), receiver (Rx), and scatterer. For simplicity, we derive our signal model under the assumption that each transmitter is operated sequentially within the coherence time of the scene. In the absence of noise 
and interference, the resulting signal model is equivalent to the phased array case, which is of interest here. For clarity of presentation, we do not consider extensions to $2 \mathrm{D}$ or $3 \mathrm{D}$ arrays in this paper.

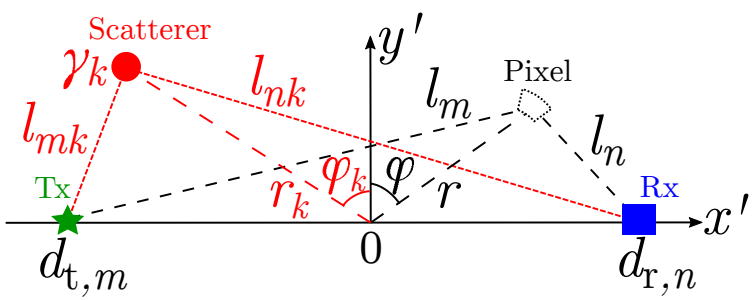

Fig. 1. Imaging in the plane with a linear array. Transmit element $m$ and receive element $n$ are located at $d_{\mathrm{t}, m}$ and $d_{\mathrm{r}, n}$. Scatterer $k$ with reflectivity $\gamma_{k}$ has polar coordinates $\left(r_{k}, \varphi_{k}\right)$, or alternatively $\left(r_{k}, v_{k}\right)$, where $v_{k}=$ $\sin \left(\varphi_{k}\right)$. The distance from the transmitter to the receiver, via the $k$ th target is $l_{n m k}=l_{m k}\left(r_{k}, v_{k}\right)+l_{n k}\left(r_{k}, v_{k}\right)$. Similarly, the distance via the pixel in focus at $(r, u)$ is $l_{n m}=l_{m}(r, u)+l_{n}(r, u)$, where $u=\sin (\varphi)$.

\section{A. Received signal}

Let $p(t)=s(t) e^{j 2 \pi c t / \lambda_{\mathrm{c}}}$ denote the transmitted pulse, where $s(t) \in \mathbb{C}$ is the complex amplitude, $\lambda_{\mathrm{c}}$ the carrier wavelength, and $c$ the propagation speed of the wave. The pulse is transmitted from the $m$ th transmitter, and scattered off the $k$ th scatterer with reflectivity $\gamma_{k} \in \mathbb{C}$. The backscattered signal is then received at the $n$th receiver after delay $\tau_{n m k}=l_{n m k} / c$, where $l_{n m k}=l_{m k}+l_{n k}$ denotes the distance from the $m$ th Tx element to the $n$th $\mathrm{Rx}$ element via the $k$ th scatterer. In the absence of clutter and noise, the Rx signal $x_{n m}$ is a superposition of the attenuated scatterer reflections:

$$
x_{n m}(t)=\sum_{k=1}^{K} \frac{g_{n m k}}{l_{n m k}} \gamma_{k} s\left(t-\tau_{n m k}\right) e^{j 2 \pi c\left(t-\tau_{n m k}\right) / \lambda_{\mathrm{c}}} .
$$

Here $g_{n m k}=g_{\mathrm{r}, n k} g_{\mathrm{t}, m k} \in \mathbb{C}$ is the two-way active element pattern in the direction of scatterer $k$, where $g_{\mathrm{r}, n k}$ and $g_{\mathrm{t}, m k}$ are the respective $\mathrm{Rx}$ and $\mathrm{Tx}$ active element patterns. The active element pattern accounts for element gain, and any non-idealities of real world sensor arrays, such as mutual coupling [20]. In (1), $g_{n m k}$ and $\gamma_{k}$ are assumed to be frequency independent, and higher order reflections are ignored.

\section{B. Signal after beamforming, image addition, and scaling}

The array is focused by delaying the received signals in (1) by $\tau_{n m}(r, u)=l_{n m}(r, u) / c$, where $r$ is the range to the pixel in focus, and $u=\sin (\varphi)$ the direction of the pixel. Furthermore, $l_{n m}=l_{n}+l_{m}$ denotes the Tx-to-pixel-to-Rx distance, with $l_{i}(r, u)=\sqrt{r^{2}+d_{i}^{2}-2 r d_{i} u}$. Signals $x_{n m}$ are then multiplied by $\mathrm{Tx} / \mathrm{Rx}$ image addition weights $w_{\mathrm{t}, m q}, w_{\mathrm{r}, n q} \in \mathbb{C}$, scaled by $2 r$ to compensate for free-space propagation loss, and summed, yielding

$$
y(t, r, u)=\sum_{q=1}^{Q} \sum_{n=1}^{N_{\mathrm{r}}} \sum_{m=1}^{N_{\mathrm{t}}} w_{\mathrm{r}, n q} w_{\mathrm{t}, m q} 2 r x_{n m}\left(t+\tau_{n m}\right) .
$$

Here $Q$ is the number of image addition components, which determines the number of transmitted pulses when Tx elements are operated coherently, as in a phased array.

\section{Reflectivity estimate after matched filtering}

Matched filtering of (2) with the transmitted pulse $p(t)$ produces an estimate of the reflectivity at $(r, u)$. The output of the matched filter sampled at $\tau=0$ is $\hat{\gamma}(r, u)=\int_{-\infty}^{\infty} p^{*}(t-$ $\tau)\left.y(t, r, u) d t\right|_{\tau=0}$, which evaluates to

$$
\hat{\gamma}(r, u)=\sum_{q=1}^{Q} \sum_{n=1}^{N_{\mathrm{r}}} \sum_{m=1}^{N_{\mathrm{t}}} w_{\mathrm{r}, n q} w_{\mathrm{t}, m q} \sum_{k=1}^{K} G_{n m k} \gamma_{k} R_{s} e^{j 2 \pi c \Delta \tau_{n m k} / \lambda_{\mathrm{c}}}
$$

where $R_{s}\left(\Delta \tau_{n m k}\right)=\left.\int_{-\infty}^{\infty} s^{*}(t-\tau) s\left(t+\Delta \tau_{n m k}\right) d t\right|_{\tau=0}$ is the autocorrelation of the transmitted baseband signal $s$, and $(\cdot)^{*}$ denotes complex conjugation. Furthermore, $\Delta \tau_{n m k}=$ $\left(l_{n m}(r, u)-l_{n m}\left(r_{k}, v_{k}\right)\right) / c$ is the difference between the focusing delay of the pixel and the round-trip delay to the $k$ th scatterer in direction $v_{k}=\sin \left(\varphi_{k}\right)$. The scaled two-way active element pattern in that same direction is denoted by $G_{n m k}=g_{n m k} \cdot 2 r / l_{n m k}$.

\section{Point spread function and co-array weight matrix}

Consider a single scatterer with unit reflectivity $\left(\gamma_{k}=1\right)$ in direction $v \in[-1,1]$ and in range focus $\left(r_{k}=r\right)$. In this case, (3) yields the spatially varying PSF, which can compactly be expressed as:

$$
\psi(r, u, v)=\operatorname{Tr}\left(\mathbf{A}_{\text {tr }}^{\mathrm{T}} \mathbf{W}\right),
$$

with $\mathbf{A}_{\mathrm{tr}}, \mathbf{W} \in \mathbb{C}^{N_{\mathrm{r}} \times N_{\mathrm{t}}}$. The two-way steering matrix in (4) is

$$
\mathbf{A}_{\mathrm{tr}}(r, u, v)=\mathbf{G} \circ \mathbf{R}_{s} \circ e^{j 2 \pi c \boldsymbol{\Delta} \boldsymbol{\tau} / \lambda},
$$

where $\circ$ denotes the Hadamard product, and $\mathbf{R}_{s}=R_{s}(\boldsymbol{\Delta} \boldsymbol{\tau})$. Function $R_{s}$ and the exponential function in (5) are applied element-wise to delay matrix $\Delta \tau$, whose $(n, m)$ th entry is $\Delta \tau_{n m}=\left(l_{n m}(r, u)-l_{n m}(r, v)\right) / c$. Similarly, the $(n, m)$ th entry of the gain matrix $\mathbf{G}$ is $G_{n m}=g_{n m}(r, v) \cdot 2 r / l_{n m}(r, v)$. The co-array weight matrix $\mathbf{W} \in \mathbb{C}^{N_{\mathrm{r}} \times N_{\mathrm{t}}}$ is given by the outer product of $Q$ receive and transmit weight vectors $\mathbf{w}_{\mathrm{r}, q}=$ $\left[w_{\mathrm{r}, 1 q}, \ldots, w_{\mathrm{r}, N_{\mathrm{r}} q}\right]^{\mathrm{T}} \in \mathbb{C}^{N_{\mathrm{r}}}$ and $\mathbf{w}_{\mathrm{t}, q}=\left[w_{\mathrm{t}, 1 q}, \ldots, w_{\mathrm{t}, N_{\mathrm{t}} q}\right]^{\mathrm{T}} \in \mathbb{C}^{N_{\mathrm{t}}}$ :

$$
\mathbf{W}=\sum_{q=1}^{Q} \mathbf{w}_{\mathrm{r}, q} \mathbf{w}_{\mathrm{t}, q}^{\mathrm{T}}
$$

The weight vectors in (6) may be obtained through the SVD of $\mathbf{W}$, in which case $Q=\operatorname{rank}(\mathbf{W}) \leq \min \left(N_{\mathrm{r}}, N_{\mathrm{t}}\right)$ [18]. Matrix $\mathbf{W}$ is in turn determined by the desired point spread function, and the sum co-array [18]. The support of the sum co-array consists of the sums of all Tx-Rx element pair positions, which in this case is the set $\left\{d_{\mathrm{t}, m}+d_{\mathrm{r}, n}\right\}_{m, n=1}^{N}$.

\section{E. Sampling the two-way steering matrix}

Subsequent sections assume that the two-way steering matrix $\mathbf{A}_{\text {tr }}$ in (5) is known for $(r, u, v)$ tuples of interest. Sampling the angular domain of the scatterer in a set of directions $\left\{v_{i}\right\}_{i=1}^{V}$ yields a set of matrices $\left\{\mathbf{A}_{\mathrm{tr}}\left(r, u, v_{i}\right)\right\}_{i=1}^{V}=$ $\left\{\mathbf{A}_{\mathrm{tr}, i}\right\}_{i=1}^{V}$ for each $(r, u)$ pair. Vectorizing and collecting these matrices into a matrix $\mathbf{A}=\left[\operatorname{vec}\left(\mathbf{A}_{\mathrm{tr}, 1}\right), \ldots, \operatorname{vec}\left(\mathbf{A}_{\mathrm{tr}, V}\right)\right]^{\mathrm{T}} \in$ $\mathbb{C}^{V \times N_{\mathrm{r}} N_{\mathrm{t}}}$, allows the PSF in (4) to be written as:

$$
\boldsymbol{\psi}=\mathbf{A v e c}(\mathbf{W}) \text {. }
$$


In practice, analytical expressions for the terms in (5) may be unavailable, and the two-way steering matrix $\mathbf{A}_{\mathrm{tr}}(r, u, v)$ must be obtained through measurements or simulations. This implies that $V$ calibration matrices of size $N_{\mathrm{r}} \times N_{\mathrm{t}}$ should be collected for each pixel $\left\{\left(r_{i}, u_{i}\right)\right\}_{i=1}^{P}$, leading to a total of $V P$ matrices, where $P$ is the number of pixels. Fortunately, this number may be substantially reduced by noting that (5) can also be written as

$$
\mathbf{A}_{\mathrm{tr}}(r, u, v)=\tilde{\mathbf{A}}_{\mathrm{tr}}(r, v) \circ \check{\mathbf{A}}_{\mathrm{tr}}(r, u) \circ \mathbf{R}_{s}(r, u, v),
$$

where the $(n, m)$ th entries of $\tilde{\mathbf{A}}_{\mathrm{tr}}(r, v)$ and $\check{\mathbf{A}}_{\mathrm{tr}}(r, u)$ in (8) are $\tilde{A}_{\mathrm{tr}, n m}(r, v)=\exp \left(-j 2 \pi l_{n m}(r, v) / \lambda_{\mathrm{c}}\right) g_{n m}(r, v) / l_{n m}(r, v)$, respectively $\check{A}_{\mathrm{tr}, n m}(r, u)=l_{n m}(r, u) \exp \left(j 2 \pi l_{n m}(r, u) / \lambda_{\mathrm{c}}\right)$. When the transmitted signal is narrowband, function $R_{s}$ is constant, and (8) becomes separable in $v$ and $u$. In this case, only $\tilde{\mathbf{A}}_{\mathrm{tr}}(r, v)$ needs to be measured, since $\check{\mathbf{A}}_{\mathrm{tr}}(r, u)$ can be precomputed for any pixel. Matrix entry $\tilde{A}_{\mathrm{tr}, n m}(r, v)$ is obtained by measuring the response of $\mathrm{Rx}$ element $n$ to a narrowband transmission from $\mathrm{Tx}$ element $m$, with a point scatterer present at $(r, v)$. If the range is sampled at a finite set of $J \ll P$ bins $\left\{r_{i}\right\}_{i=1}^{J}$, a total of $V J$ matrices of size $N_{\mathrm{r}} \times N_{\mathrm{t}}$ need to be collected.

\section{ARRAY SENSITIVITY TO NEAR FIELD EFFECTS}

As a rule of thumb, the transition between near and far field at wavelength $\lambda$ occurs when $r \propto L^{2} / \lambda$, where $L$ is the array aperture [6], [9]. The difference between the near and far field beampatterns depends on the employed element weighting [21], [22], look direction [6], and array configuration, specifically when using co-array processing [23].

The effect of the near field on the array beampattern may be understood as spatial aliasing [24], since in the near field, the plane wave assumption no longer holds, and scatterers cease to be spatially narrowband. An alternative interpretation may be given in terms of the co-array, which varies spatially for near field scatterers. Consider the path difference $c \Delta \tau_{n m}=$ $l_{n m}(r, u)-l_{n m}(r, v)$ between the pixel and a single scatterer in range focus at angle $v$. Approximating $c \Delta \tau_{n m}$ by the two leading-order terms of its Taylor expansion yields [6]

$$
c \Delta \tau_{n m} \approx\left(d_{\mathrm{r}, n}+d_{\mathrm{t}, m}+\left(d_{\mathrm{r}, n}^{2}+d_{\mathrm{t}, m}^{2}\right) \frac{v+u}{2 r}\right)(v-u) .
$$

For finite $r$, the term $\left(d_{\mathrm{r}, n}^{2}+d_{\mathrm{t}, m}^{2}\right)(v+u) /(2 r)$ in (9) can be interpreted as a perturbation to the sum co-array $d_{\mathrm{r}, n}+d_{\mathrm{t}, m}$, such that it is not necessarily uniform anymore. A sum coarray with few redundancies will be more susceptible to these perturbations, as the elements of the spatially varying co-array become non-uniformly distributed.

For example, consider the two arrays with co-located transceivers in Fig. 2. The filled Uniform Linear Array (ULA) has $N_{\mathrm{r}}=N_{\mathrm{t}}=23$ sensors, whereas the sparse MinimumRedundancy Array (MRA) [25], [26] has 11. The MRA minimizes the number of elements in the array subject to the sum co-array being a ULA. Both the ULA and MRA have the same sum co-array, therefore achieving the same far field PSF. When the inter-element spacing is half a wavelength, the physical aperture of both arrays is $L=11 \lambda_{c}$. Fig. 3 shows the spatially varying co-array of the MRA and ULA, given a single boresight scatterer $(v=0)$ at view angle $u=\sin (\pi / 4)$ and a distance of 11 baseline lengths $\left(r=121 \lambda_{\mathrm{c}}\right)$. The spatially varying co-array of the MRA is visibly less uniform than that of the ULA, because the ULA has more unique elements in its spatially varying co-array. Fig. 4 shows the PSF of the ULA and MRA, assuming ideal omnidirectional elements $\left(g_{n m}=1\right)$ and a narrowband transmitted waveform $\left(R_{s}=1\right)$. The ULA requires $Q=2$, and the MRA $Q=6$ component images to achieve the desired far field Dolph-Chebyshev beampattern [27] given by the solid red line. The dashed blue lines are the near field PSFs using the far field weights. The finite range does not affect the ULA, whereas the MRA displays clear grating lobes. These grating lobes may be mitigated by reducing the field of view, increasing the imaging range, or by adding elements to the array, as suggested by (9). Alternatively, we may compute new element weights that approximately achieve the desired PSF in the near field, as discussed next.

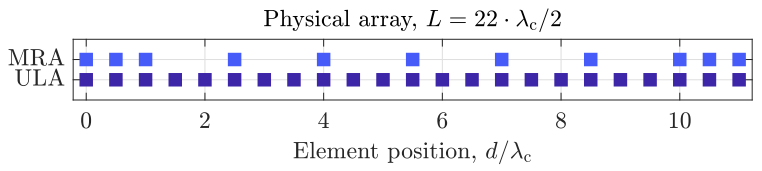

Fig. 2. MRA and ULA with aperture $L=11 \lambda_{\mathrm{c}}$. Both arrays have the same sum co-array, which is a 45 element virtual ULA with aperture $22 \lambda_{c}$.

\section{CO-ARRAY WEIGHT OPTIMIZATION PROBLEM}

The co-array weight optimization problem may be expressed as follows. For a given $(r, u)$, we seek matrix $\mathbf{W} \in$ $\mathbb{C}^{N_{\mathrm{r}} \times N_{\mathrm{t}}}$ that minimizes the discrepancy between the true PSF $\boldsymbol{\psi}(r, u, \mathbf{W}) \in \mathbb{C}^{V}$ in (7) and a desired PSF $\boldsymbol{\psi}_{\mathrm{d}} \in \mathbb{C}^{V}$. Furthermore, we constrain the rank of $\mathbf{W}$ to the desired number of component images $Q$. Choosing the $\ell_{2}$-norm as the loss function yields the non-convex optimization problem:

$$
\begin{array}{ll}
\underset{\mathbf{W} \in \mathbb{C}^{N_{\mathrm{r}} \times N_{\mathrm{t}}}}{\operatorname{minimize}} & \left\|\boldsymbol{\psi}_{\mathrm{d}}-\operatorname{Avec}(\mathbf{W})\right\|_{2} \\
\text { subject to } & \operatorname{rank}(\mathbf{W})=Q,
\end{array}
$$

which is a low-rank matrix recovery problem [28]. Note that $\operatorname{rank}(\mathbf{W})$ may be minimized when $\operatorname{Avec}(\mathbf{W})=\psi_{\mathrm{d}}$, which was the case considered in our previous work [19]. However, Avec $(\mathbf{W})=\boldsymbol{\psi}_{\mathrm{d}}$ cannot be guaranteed in (P1), since knowledge of the spatially varying co-array is not assumed. Nevertheless, (P1) can be solved offline and in parallel for each $(r, u)$ of interest, provided that the non-convexity of the problem is addressed first.

\section{PROpOSED SOLUTIONS}

This section proposes two convex relaxations to (P1). The first solution modifies the rank constrained problem to a Tikhonov regularized least squares (LS) problem. The resulting semi closed-form expression is fast to evaluate, but typically results in a high number of component images $Q$, which may unnecessesarily increase the image acquisition time. The second solution relaxes the rank constraint to its closest convex surrogate, the nuclear norm [29]. This semidefinite program (SDP) allows a trade-off between $Q$ and approximation error, at the expense of a higher computational cost. Note that non-convex approaches have also been proposed for solving (P1). For example, alternating minimization solves a biconvex problem, which essentially involves iteratively solving two LS problems is succession [28], [30]. Unfortunately, such algorithms have no guarantees of convergence to global optima. 


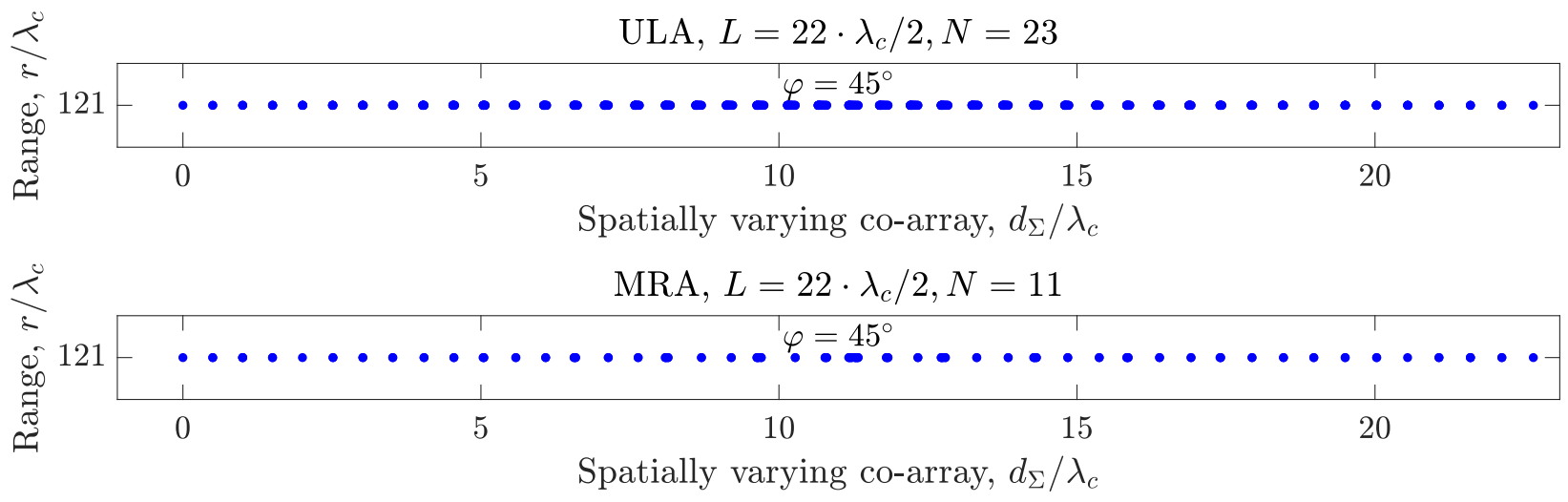

Fig. 3. Spatially varying co-array of ULA and MRA for single boresight scatterer at finite range $\left(r=121 \lambda_{\mathrm{c}}, u=\sin (\pi / 4), v=0\right)$. Due to having fewer physical elements than the ULA, the MRA also has a less uniform spatially varying co-array (especially around the midpoint). Note that both arrays have the same uniform (far field) sum co-array.

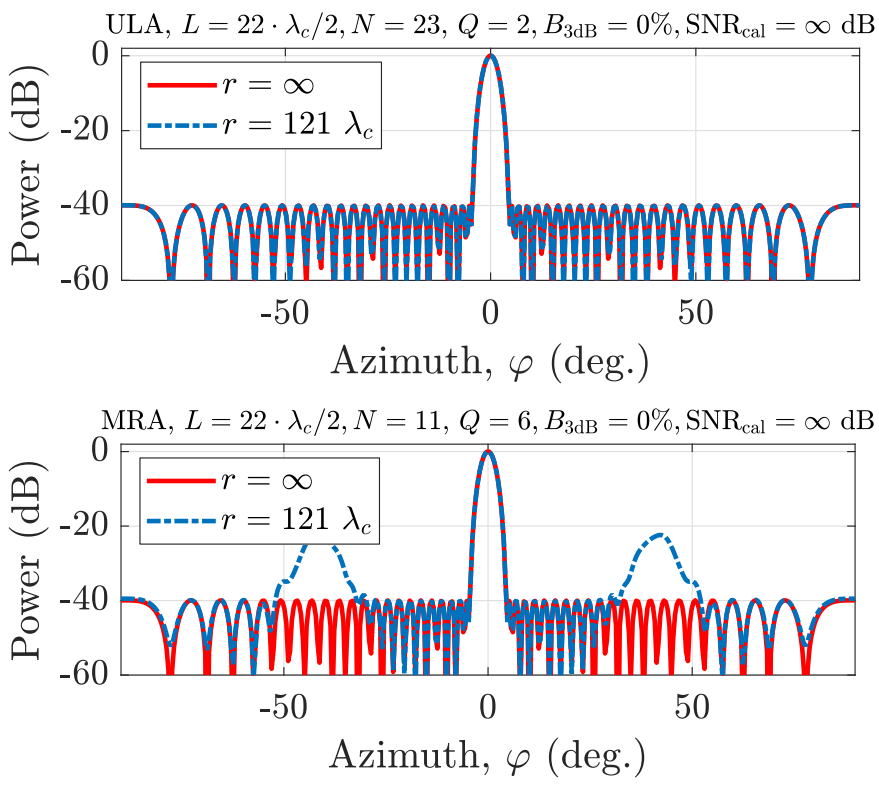

Fig. 4. PSF of ULA (top) and MRA (bottom) in the far field (solid red line) and near field (dashed blue line) using far field weights (Dolph-Chebyshev sum co-array weighting with $-40 \mathrm{~dB}$ side lobes). The MRA suffers from grating lobes in the near field, since its spatially varying co-array is non-uniform.

\section{A. Least squares approximation}

Squaring the objective function in (P1), and replacing the rank constraint by a Frobenius norm regularizer $\beta\|\mathbf{W}\|_{\mathrm{F}}^{2}, \beta>0$ yields the convex unconstrained optimization problem:

$$
\underset{\mathbf{W} \in \mathbb{C}^{N_{\mathrm{r}} \times N_{\mathrm{t}}}}{\operatorname{minimize}}\left\|\boldsymbol{\psi}_{\mathrm{d}}-\operatorname{Avec}(\mathbf{W})\right\|_{2}^{2}+\beta\|\mathbf{W}\|_{\mathrm{F}}^{2} .
$$

Problem (P2) admits a unique, semi closed-form solution given by the diagonally loaded LS, or ridge regression solution:

$$
\operatorname{vec}(\mathbf{W})=\left(\mathbf{A}^{\mathrm{H}} \mathbf{A}+\beta \mathbf{I}\right)^{-1} \mathbf{A}^{\mathrm{H}} \boldsymbol{\psi}_{\mathrm{d}} .
$$

Matrix $\mathbf{A}^{\mathrm{H}} \mathbf{A} \in \mathbb{C}^{N_{\mathrm{r}} N_{\mathrm{t}} \times N_{\mathrm{r}} N_{\mathrm{t}}}$ may be ill-conditioned, especially if $V<N_{\mathrm{r}} N_{\mathrm{t}}$. In practice, the diagonal loading parameter $\beta$ has to be tuned manually so that it is as small as possible, yet does not cause numerical instability or overfit the solution to noisy measurements. Evaluating (10) requires $\mathcal{O}\left(N_{\mathrm{r}}^{3} N_{\mathrm{t}}^{3}\right)$ arithmetic operations, assuming $V \ngtr N_{\mathrm{r}} N_{\mathrm{t}}$.

\section{B. Nuclear norm relaxation}

Replacing the rank operator in (P1) with the nuclear norm, the tightest convex relaxation [29], leads to the convex unconstrained optimization problem

$$
\underset{\mathbf{W} \in \mathbb{C}^{N_{\mathrm{r}} \times N_{\mathrm{t}}}}{\operatorname{minimize}}\left\|\boldsymbol{\psi}_{\mathrm{d}}-\operatorname{Avec}(\mathbf{W})\right\|_{2}+\alpha\|\mathbf{W}\|_{*},
$$

where $\alpha>0$ is again a regularization parameter. Selecting the value of $\alpha$ is not a trivial problem, and one often has to resort to heuristics, such as trial-and-error, to find a value that works satisfactorily. Problem (P3) may be expressed as an SDP with an $\left(N_{\mathrm{r}}+N_{\mathrm{t}}\right) \times\left(N_{\mathrm{r}}+N_{\mathrm{t}}\right)$ PSD matrix of auxiliary variables [29]. This SDP can be solved by interiorpoint methods using roughly $\mathcal{O}\left(N_{\mathrm{r}}^{2} N_{\mathrm{t}}^{2}\left(N_{\mathrm{r}}+N_{\mathrm{t}}\right)^{2.5}\right)$ arithmetic operations, assuming $V \propto N_{\mathrm{r}} N_{\mathrm{t}}$ [31]. We use the Matlab [32] toolbox CVX [33] with SDPT3 [34] to solve (P3) in our subsequent simulations. However, faster algorithms are also readily available for problems closely related to (P3). Examples include both proximal [28], and greedy methods [35]. These algorithms have the complexity of SVD, i.e., $\mathcal{O}\left(N_{\mathrm{r}}^{3} N_{\mathrm{t}}^{3}\right)$, provided that the number of iterations is kept low.

\section{NUMERICAL EXPERIMENTS}

In this section, we numerically demonstrate the performance of the proposed solutions (P2) and (P3) using the arrays in Fig. 2. First however, we define the cumulative energy: $p(Q)=\left(\sum_{q=1}^{Q} \sigma_{q}\right) /\left(\sum_{q=1}^{\min \left(N_{\mathrm{r}}, N_{\mathrm{t}}\right)} \sigma_{q}\right)$, where $\sigma_{1} \geq$ $\sigma_{2} \geq \ldots \geq \sigma_{Q} \geq \ldots \geq \sigma_{\min \left(N_{\mathrm{r}}, N_{\mathrm{t}}\right)}$ are the singular values of matrix $\mathbf{W}$ sorted in descending order. We require that the $Q$ largest singular values of $\mathbf{W}$ explain at least $99.99 \%$ of its variance, i.e., $p(Q) \geq 0.9999$. Second, since $Q$ may vary from pixel to pixel, we also compute its average value, $\bar{Q}=\sum_{i=1}^{P} Q\left(r_{i}, u_{i}\right) / P$, where $P$ is the number of pixels.

\section{A. Near field compensated PSF}

Let us revisit the narrowband PSF of the MRA at range $r=121 \lambda_{\mathrm{c}}$ considered in section III. We assume perfect knowledge of $\mathbf{A}_{\mathrm{tr}}(r, u, v)$ in $V=100$ directions $v \in\left\{\sin \left(\varphi_{i}\right)\right\}_{i=1}^{100}$. Angle $\varphi_{i}$ is uniformly sampled between $-\pi / 2$ and $\pi / 2$, which requires collecting 100 calibration matrices of size $11 \times 11$. 
Fig. 5(a) shows the near field PSF using the LS solution (10). Grating lobes are visibly suppressed compared to Fig. 4, although the average number of component images has increased from $\bar{Q}=6$ to 11 . Fig. 5(b) shows the PSF obtained by solving the nuclear norm regularized problem (P3). Fig. 5(b) is practically indistinguishable from Fig. 5(a), despite requiring $19 \%$ fewer component images on average, as $\bar{Q}=9$.

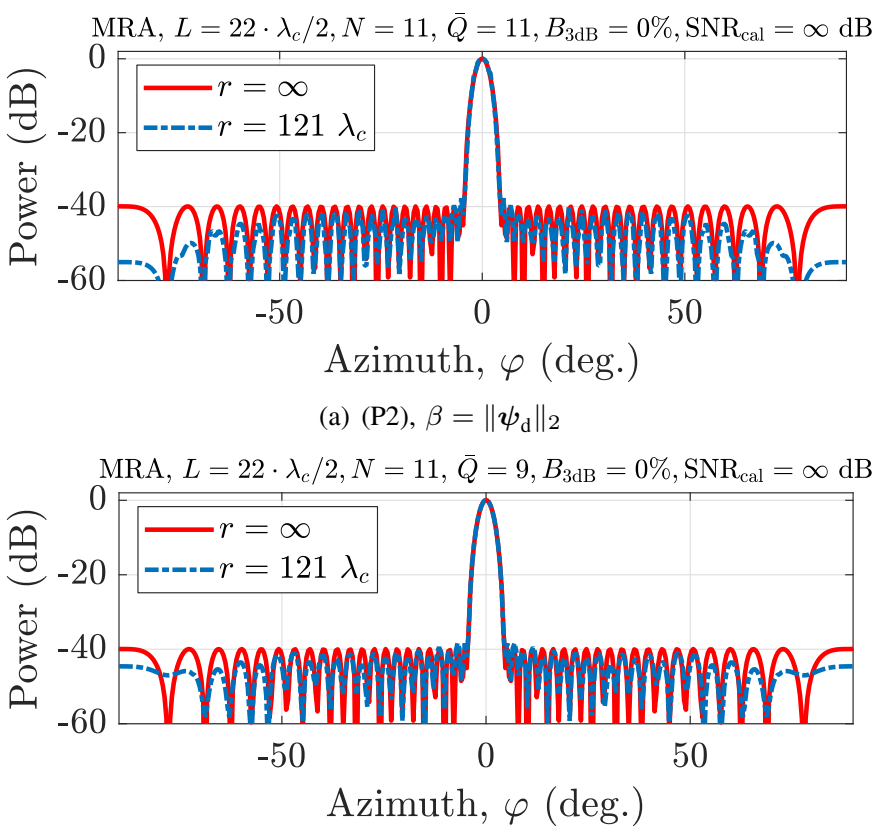

(b) (P3), $\alpha=10\left\|\boldsymbol{\psi}_{\mathrm{d}}\right\|_{2}$

Fig. 5. Near field PSF of MRA using weights solving the (a) LS problem in (P2), and (b) nuclear norm regularized problem in (P3). Grating lobes in Fig. 4 are suppressed at the expense of an increase in the average number of component images: (a) $\bar{Q}=11$, (b) $\bar{Q}=9$, compared to $\bar{Q}=6$ in Fig. 4 .

\section{B. Imaging with noisy near field calibration measurements}

Consider three unit reflectivity scatterers at coordinates $(r, \varphi) \in\left\{\left(109 \lambda_{\mathrm{c}},-\pi / 4\right),\left(121 \lambda_{\mathrm{c}}, 0\right),\left(133 \lambda_{\mathrm{c}}, \pi / 4\right)\right\}$ with respect to the center point of the array. The transmitted waveform is a square pulse lasting 10 carrier frequency cycles. Such a signal has a relative $3 \mathrm{~dB}$ bandwidth of approximately $9 \%$. The two-way steering matrix is sampled using a narrowband signal of wavelength $\lambda_{\mathrm{c}}$, such that only $\tilde{\mathbf{A}}_{\mathrm{tr}}(r, v)$ in $(8)$ is measured. The measurements are contaminated by complex additive white Gaussian noise at the receiver, where the SNR of a single sensor is $30 \mathrm{~dB}$. The imaging process itself is assumed noiseless, as in (3), in order to highlight the effects of calibration errors. The calibration grid is sampled uniformly at $V=100$ angles, and a single range $r=121 \lambda_{\mathrm{c}}$. The range of the imaging grid is uniformly sampled at 100 points between $96 \lambda_{\mathrm{c}}$ and $146 \lambda_{\mathrm{c}}$, and the look direction $u$ at 200 points between $-\pi / 2$ and $\pi / 2$. Optimization problems (P2) and (P3) are thus solved for $P=200 \times 100$ pixels.

Fig. 6(a) and 6(b) show the images produced by the ULA and MRA using far field weights. As expected, the MRA suffers from grating lobes, which do not affect the ULA. Fig. 6(c) and 6(d) show the same scene imaged by the MRA using weights obtained by solving (P2), respectively (P3). Near field compensation effectively suppresses grating lobes over the whole imaging range, despite using noisy narrowband calibration measurements from a single range. The nuclear norm formulation again produces a solution with a lower average number of component images than $\operatorname{LS}(\bar{Q}=9$ in Fig. 6(d) compared to $\bar{Q}=11$ in Fig. 6(c)).

\section{CONCLUSION}

This paper considered active near field imaging of coherent scatterers using sparse linear phased arrays. We demonstrated that sparse arrays using image addition are susceptible to grating lobes in the near field, due to the non-uniformity of the spatially varying (sum) co-array. In order to address this issue, we proposed a novel near field compensation method applicable to sum co-array processing using image addition. The method adapts the array weights to the imaging range and direction by trying to match a desired PSF with support on the sum co-array. A constraint was also added for keeping the number of component image additions low, such that the acquisition time of the image is minimized. Our problem formulation takes the form of a rank-constrained non-convex optimization problem, which uses samples of the array's near field response. We proposed two convex solutions to this problem: a Tikhonov regularized LS approximation, and an SDP relaxation using the nuclear norm. These solutions were evaluated in simulations, which demonstrated that the proposed approaches mitigate near field effects, even when calibration measurements are noisy and only available at a limited number of ranges.

\section{REFERENCES}

[1] R. T. Hoctor and S. A. Kassam, "The unifying role of the coarray in aperture synthesis for coherent and incoherent imaging," Proceedings of the IEEE, vol. 78, no. 4, pp. 735-752, Apr 1990.

[2] - "High resolution coherent source location using transmit/receive arrays," IEEE Transactions on Image Processing, vol. 1, no. 1, pp. 88100, Jan 1992.

[3] P. Pal and P. P. Vaidyanathan, "Nested arrays: A novel approach to array processing with enhanced degrees of freedom," IEEE Transactions on Signal Processing, vol. 58, no. 8, pp. 4167-4181, Aug 2010.

[4] R. A. Haubrich, "Array design," Bulletin of the Seismological Society of America, vol. 58, no. 3, pp. 977-991, 1968.

[5] J. Li and P. Stoica, MIMO radar signal processing. John Wiley \& Sons, 2009.

[6] R. J. Kozick and S. A. Kassam, "Synthetic aperture pulse-echo imaging with rectangular boundary arrays," IEEE Transactions on Image Processing, vol. 2, no. 1, pp. 68-79, Jan 1993.

[7] F. Ahmad and S. A. Kassam, "Coarray analysis of the wide-band point spread function for active array imaging," Signal Processing, vol. 81, no. 1, pp. 99-115, 2001.

[8] L. He and S. Kassam, "Near-range point spread function coarray analysis for array imaging," IEEE Transactions on Aerospace and Electronic Systems, vol. 51, no. 1, pp. 397-404, January 2015.

[9] B. Steinberg, Principles of aperture and array system design: Including random and adaptive arrays. New York, Wiley-Interscience, 1976.

[10] F. Khalil, J. P. Jullien, and A. Gilloire, "Microphone array for sound pickup in teleconference systems," J. Audio Eng. Soc, vol. 42, no. 9, pp. 691-700, 1994.

[11] T. D. Abhayapala, R. A. Kennedy, and R. C. Williamson, "Farfield array weight redesign for nearfield beamforming," http://users.cecs.anu.edu. au/ thush/publications/p98_07.pdf, 1998, accessed: 2018-07-25.

[12] R. A. Kennedy, T. D. Abhayapala, and D. B. Ward, "Broadband nearfield beamforming using a radial beampattern transformation," IEEE Transactions on Signal Processing, vol. 46, no. 8, pp. 2147-2156, Aug 1998. 
[13] R. A. Kennedy, D. B. Ward, and T. D. Abhayapala, "Nearfield beamforming using radial reciprocity," IEEE Transactions on Signal Processing, vol. 47, no. 1, pp. 33-40, Jan 1999.

[14] H. Lebret and S. Boyd, "Antenna array pattern synthesis via convex optimization," IEEE Transactions on Signal Processing, vol. 45, no. 3, pp. 526-532, Mar 1997.

[15] S. E. Nordholm, V. Rehbock, K. L. Tee, and S. Nordebo, "Chebyshey optimization for the design of broadband beamformers in the near field," IEEE Transactions on Circuits and Systems II: Analog and Digital Signal Processing, vol. 45, no. 1, pp. 141-143, Jan 1998.

[16] S. Doclo and M. Moonen, "Design of far-field and near-field broadband beamformers using eigenfilters," Signal Processing, vol. 83, no. 12, pp. $2641-2673,2003$.

[17] H. Chen, W. Ser, and J. Zhou, "Robust nearfield wideband beamformer design using worst case mean performance optimization with passband response variance constraint," IEEE Transactions on Audio, Speech, and Language Processing, vol. 20, no. 5, pp. 1565-1572, July 2012.

[18] R. J. Kozick and S. A. Kassam, "Linear imaging with sensor arrays on convex polygonal boundaries," IEEE Transactions on Systems, Man, and Cybernetics, vol. 21, no. 5, pp. 1155-1166, Sep 1991.

[19] R. Rajamäki and V. Koivunen, "Sparse array imaging using low-rank matrix recovery," in Seventh IEEE International Workshop on Computational Advances in Multi-Sensor Adaptive Processing (CAMSAP 2017), Curaçao, Dutch Antilles, December 2017.

[20] D. M. Pozar, "The active element pattern," IEEE Transactions on Antennas and Propagation, vol. 42, no. 8, pp. 1176-1178, Aug 1994.

[21] P. Hacker and H. Schrank, "Range distance requirements for measuring low and ultralow sidelobe antenna patterns," IEEE Transactions on Antennas and Propagation, vol. 30, no. 5, pp. 956-966, September 1982.

[22] R. Hansen, "Measurement distance effects on low sidelobe patterns," IEEE Transactions on Antennas and Propagation, vol. 32, no. 6, pp 591-594, June 1984

[23] R. Rajamäki and V. Koivunen, "Symmetric sparse linear array for active imaging," in Tenth IEEE Sensor Array and Multichannel Signal Processing Workshop (SAM 2018), Sheffield, UK, July 2018, pp. 46-50.

[24] T. D. Abhayapala, R. A. Kennedy, and R. C. Williamson, "Spatial aliasing for near-field sensor arrays," Electronics Letters, vol. 35, no. 10, pp. 764-765, May 1999.

[25] A. Moffet, "Minimum-redundancy linear arrays," IEEE Transactions on Antennas and Propagation, vol. 16, no. 2, pp. 172-175, Mar 1968.

[26] J. Kohonen, "A meet-in-the-middle algorithm for finding extremal restricted additive 2-bases," Journal of Integer Sequences, vol. 17, no. 6 , 2014.

[27] C. L. Dolph, "A current distribution for broadside arrays which optimizes the relationship between beam width and side-lobe level," Proceedings of the IRE, vol. 34, no. 6, pp. 335-348, June 1946.

[28] M. A. Davenport and J. Romberg, "An overview of low-rank matrix recovery from incomplete observations," IEEE Journal of Selected Topics in Signal Processing, vol. 10, no. 4, pp. 608-622, June 2016.

[29] M. Fazel, "Matrix rank minimization with applications," Ph.D. dissertation, Stanford University, 2002.

[30] P. Jain, P. Netrapalli, and S. Sanghavi, "Low-rank matrix completion using alternating minimization," in Proceedings of the forty-fifth annual ACM symposium on Theory of computing. ACM, 2013, pp. 665-674

[31] Y. Nesterov, Introductory lectures on convex optimization: A basic course. Springer Science \& Business Media, 2013, vol. 87, pp. 218219.

[32] MATLAB, version 9.4.0.813654 (R2018a). Natick, Massachusetts: The MathWorks Inc., 2018.

[33] M. Grant and S. Boyd, "CVX: Matlab software for disciplined convex programming, version 2.1," http://cvxr.com/cvx, Mar. 2014.

[34] K. C. Toh, M. J. Todd, and R. H. Tütüncü, "SDPT3 a Matlab software package for semidefinite programming," Optimization Methods and Software, vol. 11, no. 1-4, pp. 545-581, 1999.

[35] Z. Wang, M.-J. Lai, Z. Lu, W. Fan, H. Davulcu, and J. Ye, “Orthogonal rank-one matrix pursuit for low rank matrix completion," SIAM Journal on Scientific Computing, vol. 37, no. 1, pp. A488-A514, 2015.

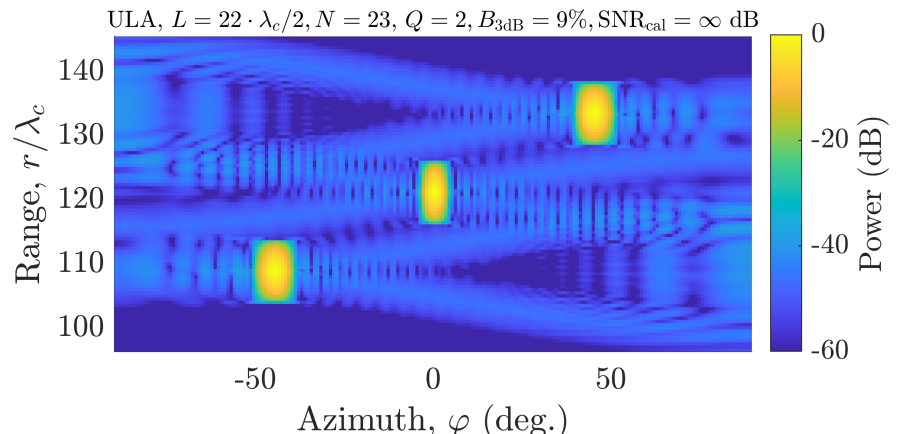

(a) ULA, far field weights

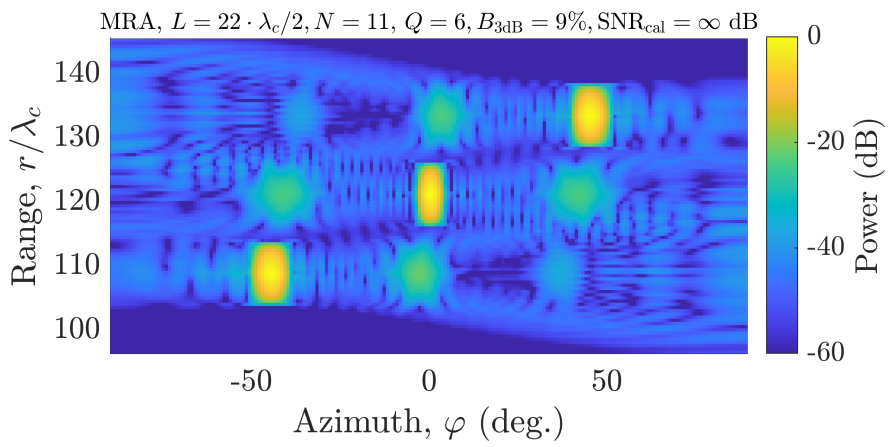

(b) MRA, far field weights

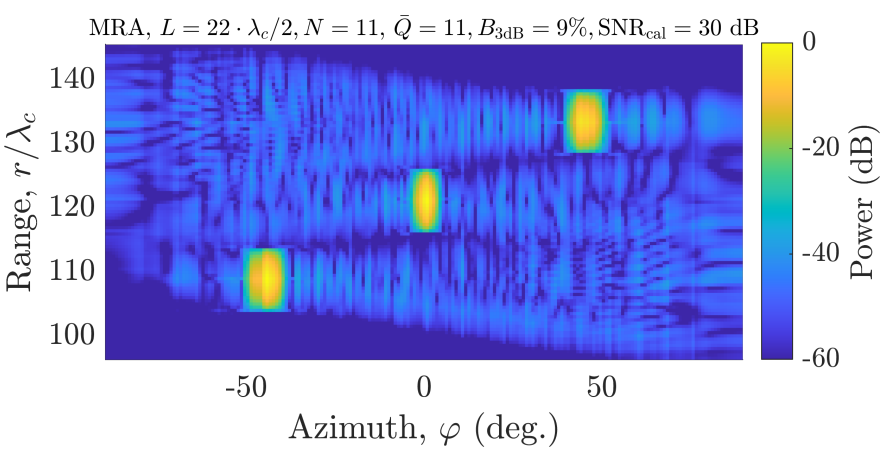

(c) MRA, near field weights solving (P2), $\beta=\left\|\boldsymbol{\psi}_{\mathrm{d}}\right\|_{2}$

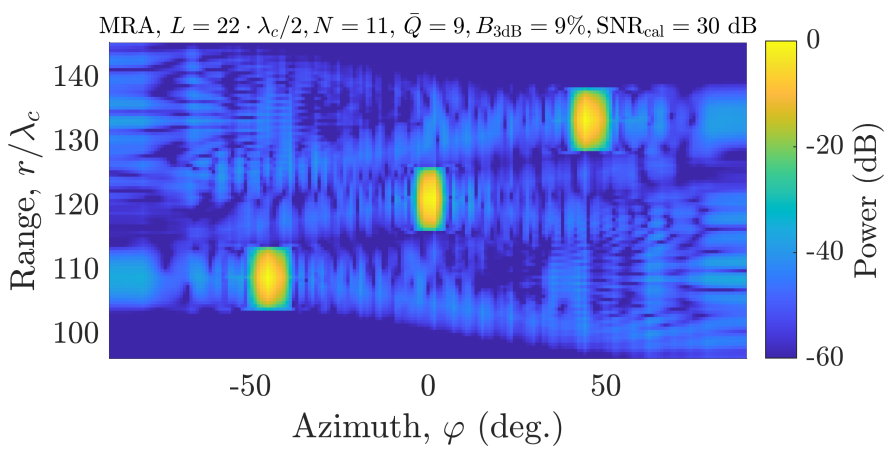

(d) MRA, near field weights solving (P3), $\alpha=10\left\|\psi_{\mathrm{d}}\right\|_{2}$

Fig. 6. Images of three coherent point reflectors at a distance of 11 array baselines. When far field weights are used, the (b) MRA is severely affected grating lobes in comparison to the (a) ULA. Both (c) LS and (d) nuclear norm based near field compensation effectively suppresses these grating lobes. 